\title{
Survival analysis of African catfish and Nile tilapia briefly exposed to complex pesticide mixtures
}

Kingsley C. Kanu ${ }^{1,2^{*}}$ (D, Adebayo A. Otitoloju² and Nnamdi H. Amaeze ${ }^{2}$

\begin{abstract}
Background: Pulse exposures are the consequences of the intermittent release of pollutants in the environment. Brief exposure of aquatic organisms to high concentrations of pesticides simultaneously occurs, particularly in small watercourses during high flows. The effects of pulse exposure often include effects occurring during and after the exposure. Despite this, routine toxicity tests procedures often ignore brief exposure scenarios and the role of time in toxicity. We conducted a pulse toxicity test by briefly exposing African catfish and Nile tilapia fingerlings to pesticide mixtures of atrazine, mancozeb, chlorpyrifos, and lambda-cyhalothrin. The study aimed to estimate pesticide mixture interaction in pulse-exposed fish and elucidate the influence of species differences on the response of fish to the pesticide mixture.

Results: Despite the similarity in fingerlings weight, African catfish had a significantly higher survival probability than Nile tilapia after exposure to atrazine-mancozeb mixture. However, the survival probability of African catfish and Nile tilapia fingerlings were similar after exposure to atrazine-chlorpyrifos, atrazine-lambda cyhalothrin, mancozebchlorpyrifos, mancozeb-lambda cyhalothrin, chlorpyrifos-lambda cyhalothrin, and quaternary mixture $(p>0.05)$. The survival probability of exposed fingerlings was significantly lower for continuous than pulse exposure to the mixtures $(p<0.01)$. Nevertheless, the survival probability of $60 \mathrm{~min}$ of pulse exposure to $13.49 \mathrm{mg} / \mathrm{L}$ mancozeb-lambda cyhalothrin was similar to continuous exposure for $96 \mathrm{~h}$. Atrazine-mancozeb, atrazine-chlorpyrifos, atrazine-lambda cyhalothrin, mancozeb-chlorpyrifos, mancozeb-lambda cyhalothrin, and the quaternary pesticide mixture were antagonists in African catfish but not in Nile tilapia. At the same time, chlorpyrifos-lambda-cyhalothrin was antagonistic in Nile tilapia but not African catfish.
\end{abstract}

Conclusions: Pesticide mixture interaction was antagonist but specie-dependent. Innate intrinsic and extrinsic deterministic factors and, to a limited extent, stochastic processes may have influenced the survival probability of African catfish, and Nile tilapia pulsed exposed to complex pesticide mixtures. Pulse toxicity assessment using survival analysis is relevant in ecotoxicology as it enables the study of factors that can influence pulse toxicity.

Keywords: Survival analysis, Pulse-exposure, Pesticide mixture, Hazard ratio, African catfish, Tilapia

\footnotetext{
*Correspondence: kanukc@gmail.com

1 Department of Environmental Management and Toxicology, Michael

Okpara University of Agriculture, Umudike, Nigeria

Full list of author information is available at the end of the article
}

\section{Background}

Pulse exposure involves one isolated, brief exposure. Repeated pulse exposure involves more than one sole short exposure while fluctuating exposure is continuous exposure to varying toxicant concentrations (Dennis et al., 2012).

Surface waters are natural sinks for pesticide mixtures. Pesticides enter the aquatic ecosystem through 
direct application, spray drift, runoff from agricultural fields, dry and wet deposition, and urban and industrial discharges (Sumon et al., 2018). Entrance of pesticides into the aquatic environment often occurs intermittently (Andersen et al., 2006) with aquatic organisms, including fish, exposed in pulse or repeated pulse to the pesticides. Pulse exposure time could vary from a few minutes to several hours depending on the properties of the pesticide and the characteristics of the water body (Cold \& Forbes, 2004). Pesticides in streams typically reach high levels for short periods and decrease to very low or undetectable levels. Although maximum concentrations are present for only a short time before dilution, aquatic life is exposed to high pesticide concentrations during peak periods capable of inducing toxic effects and death in early life stages (Ewing, 1999; Peterson et al., 2001).

Traditionally, ecotoxicology assessed the toxicity of one or a mixture of pesticide compounds using continuous exposure setups. The constant exposure approach is at variance with the pulse or repeated pulse exposure (Dennis et al., 2012). Environmental pesticide exposure is non-constant, particularly for freshwater compartments (Chèvre \& Vallotton, 2013). Furthermore, other than concentration and exposure time, other factors could also influence pesticide toxicity. These factors include the presence of other chemicals (mixture), species difference, size, and sex of the test organism. The effect of a covariate on the toxicity of a chemical is expressed using different indices. The median time to death $\left(\mathrm{LT}_{50}\right)$, the concentration at which $50 \%$ mortality occurs $\left(\mathrm{LC}_{50}\right)$, or the hazard function (the instantaneous probability of dying) are examples of such indices. A change in any one of these, not just the difference in $\mathrm{LC}_{50}$ that is typically used, can express the effect of a covariate (Dixon \& Newman, 1991).

Atrazine, mancozeb, chlorpyrifos, and lambdacyhalothrin are commonly used agricultural pesticides (WAAPP, 2013; Wang et al., 2016). Atrazine is a pre and post-emergence herbicide used to control annual broadleaved weeds and annual grass weeds in yam, maize, sorghum, and sugarcane (Tomlin, 1994). The half-life of atrazine in the surface water is greater than 100 days at $20{ }^{\circ} \mathrm{C}$. Photolysis, microbial degradation via $\mathrm{N}$-dealkylation, and hydrolysis of the chloro substituent are pathways for atrazine degradation (WHO, 2003). Mancozeb is a fungicide used to control fungi affecting potato, tomato, citrus, banana, onions, corn, wheat, and mangoes (Kumar et al., 2018). Mancozeb half-life in water is less than 2 days in which it breaks down to release ethylene bis isothiocyanate sulfide (EBIS), which is subsequently converted into ethylene bis isothiocyanate (EBI) by the action of UV light (Raghavendra et al., 2020). Chlorpyrifos is an organophosphate insecticide, which adsorbs onto suspended solids and sediment. Its solubility in water varies between 0.39 and $1.4 \mathrm{mg} / \mathrm{L}$ at a temperature range of 19.5 to $25^{\circ} \mathrm{C}$ (NCBI, 2021). The hydrolysis halflives at $25^{\circ} \mathrm{C}$ in aqueous buffers at $\mathrm{pH} 5, \mathrm{pH} 7$, and $\mathrm{pH} 9$ were 72,72 , and 16 days. The reported half-life in natural water was 24.5 days (NCBI, 2021). Chlorpyrifos is neurotoxic. It inhibits the breakdown of acetylcholine (ACh), a neurotransmitter, by binding to the enzyme acetylcholinesterase. Lambda-cyhalothrin (alpha-cyano-3-phenoxybenzyl (Z)-(1RS)-cis 3-(2-chloro-3,3,3-trifluoro propenyl)-2,2-dimethyl cyclopropane carboxylate) is a pyrethroid insecticide. Its solubility in purified water is $5 \times 10^{-3} \mathrm{mg} / \mathrm{L}$, while it is $4 \times 10^{-3} \mathrm{mg} / \mathrm{L}$ in buffered water. Lambda-cyhalothrin is neurotoxic, causing swift paralysis and death to an insect by preventing the closure of the voltage-gated sodium channels in axonal membranes.

Survival analysis is a suitable technique utilized to study pulse exposure and factors that modify the toxicity of pesticides (Kanu et al., 2021a) and other pollutants (Dixon \& Newman, 1991; Newman \& Aplin, 1992; Newman \& McCloskey, 1996). Survival analysis starts with the times individuals die. In contrast, standard toxicity testing analysis begins with data on the percentage of individuals that die after a specific period (e.g., 24, 48, 72, or 96 h) (Dixon \& Newman, 1991). Limited research has applied survival analysis to study the pulse toxicity of pesticide mixtures. We had previously used survival analysis to study the influence of pulse length, fish weight, length, and condition factor on the toxicity of atrazine, mancozeb, chlorpyrifos, and lambda-cyhalothrin in African catfish and Nile tilapia (Kanu et al., 2021a). This study aimed to estimate pesticide mixture interaction in pulseexposed fish and elucidate the influence of species difference and size on the response of fish to pesticide mixture pulse exposure.

\section{Methods \\ Study design}

Fish survival was studied after exposing fingerlings to high concentrations of complex pesticide mixtures for short time intervals. Survival analysis was used to study the effect of pesticide mixture, species difference, fingerling weight, length, and condition factor on the survival of the fingerling was after brief exposure to agricultural pesticide mixtures.

\section{Collection and acclimatization of fingerlings}

African catfish, Clarias gariepinus and Nile tilapia, Oreochromis niloticus fingerlings are commonly cultivated in fish farms and widely distributed in the natural waters of Nigeria. We obtained the fingerlings used in the study from a private fish farm and transported them to the laboratory in the morning. As reported in our previous 
study, the fish were handled and acclimatized to laboratory conditions (Kanu et al., 2021a).

\section{Test pesticides}

In Nigeria, farmers commonly use the pesticides utilized in the study (WAAPP, 2013). They include; Atraz 50FW, a herbicide containing $500 \mathrm{~g} / \mathrm{L}$ atrazine active ingredient; Z-force, a fungicide containing $80 \%$ mancozeb active ingredients; Attacke, a pyrethroid insecticide containing 2.5\% lambda-cyhalothrin active ingredient; and Chloview, an organophosphate insecticide containing $40 \%$ chlorpyrifos active ingredient.

\section{Pesticides mixture}

Stock solutions of atrazine, chlorpyrifos, and lambdacyhalothrin were prepared by making up one millilitre $(1 \mathrm{ml})$ of the pesticides to one litre $(1 \mathrm{~L})$. Mancozeb stock solution was prepared by mixing one gram $(1 \mathrm{~g})$ of the pesticide powder with one litre (1L) of water.

Stock solutions of the complex mixtures were prepared based on the ratio of the $96 \mathrm{~h} \mathrm{LC}_{50}$ of the single pesticides (established in an earlier study as atrazine- $17.463 \mathrm{mg} / \mathrm{L}$, mancozeb-24.383 mg/L, chlorpyrifos $0.515 \mathrm{mg} / \mathrm{L}$, and lambda-cyhalothrin $(0.434 \mu \mathrm{g} / \mathrm{L})$. Stock concentration and the ratio of the active ingredients in the mixture are;

\begin{tabular}{lll}
\hline Atrazine: Mancozeb & $2.39 \mathrm{~g} / \mathrm{L}$ & $(1: 1.39)$ \\
Atrazine: Chlorpyrifos & $3.495 \mathrm{~g} / \mathrm{L}$ & $(33.95: 1)$ \\
Mancozeb: Chlorpyrifos & $4.84 \mathrm{~g} / \mathrm{L}$ & $(47.35: 1)$ \\
Atrazine: Lambda cyhalothrin & $4.0301 \mathrm{~g} / \mathrm{L}$ & $(40.28: 0.001)$ \\
Mancozeb: Lambda cyhalothrin & $5.6201 \mathrm{~g} / \mathrm{L}$ & $(56.18: 0.001)$ \\
Lambda cyhalothrin: Chlorpyrifos & $0.1188 \mathrm{~g} / \mathrm{L}$ & $(0.001: 1.18)$ \\
Atrazine: Mancozeb: Chlorpyrifos: & $9.7651 \mathrm{~g} / \mathrm{L}$ & $(1.299: 0.360: 0.0132: 0.001)$ \\
Lambda cyhalothrin: & & \\
\hline
\end{tabular}

The nominal test concentrations used in the study were,

\begin{tabular}{ll}
\hline Atrazine: Mancozeb & $14.34 \mathrm{mg} / \mathrm{L}$ \\
Atrazine: Chlorpyrifos & $12.58 \mathrm{mg} / \mathrm{L}$ \\
Atrazine: Lambda cyhalothrin & $29.02 \mathrm{mg} / \mathrm{L}$ \\
Mancozeb: Chlorpyrifos & $7.74 \mathrm{mg} / \mathrm{L}$ \\
Mancozeb: Lambda cyhalothrin & $13.48 \mathrm{mg} / \mathrm{L}$ \\
Chlorpyrifos: Lambda cyhalothrin & $0.57 \mathrm{mg} / \mathrm{L}$ \\
Atrazine: Mancozeb: Chlorpyrifos: Lambda cyhalothrin & $11.72 \mathrm{mg} / \mathrm{L}$ \\
\hline
\end{tabular}

These concentrations were the maximal concentration used in a previous acute toxicity study of the pesticides using C. gariepinus, which caused maximal effects after continuous exposure for $96 \mathrm{~h}$. They were the maximum worst-case concentration (Kanu et al., 2021a).

\section{Pulse toxicity test}

All toxicity tests were performed following established procedure (APHA, 1985), except in the manner of exposure. In separate experiments, ten C. gariepinus and $O$. niloticus fingerlings were exposed in duplicates to the pesticide mixtures for 15, 30, 45, and $60 \mathrm{~min}$, and continuously for $96 \mathrm{~h}$ (similar to standard acute toxicity test). After the brief exposure, the fish were rinsed, transferred to clean water, and monitored for $96 \mathrm{~h}$. Mortality and time-to-death were checked and recorded every hour for both the pulsed and continuously exposed fish. The absence of movement and lack of response to gentle prodding established mortality. The weight and length of dead fishes were recorded, and fingerlings still alive after 96-h post-observation period were censored while their length and weight were also measured. Condition factor (CF) was computed using the equation below (Busacker et al., 1990):

$$
\mathrm{CF}=\frac{W}{L^{3}} \times 100
$$

$W$ and $L$ are the wet fish weight (g) and fish length $(\mathrm{cm})$, respectively.

\section{Estimation of complex pesticide mixture interaction using survival graph}

We used the survival graph to estimate pesticide mixture interaction. The survival probability of fish pulse exposed to a single pesticide was compared with fish pulse exposed to a pesticide mixture containing the single pesticide. Thus, we compared the survival probability of fingerlings pulse exposed to $24 \mathrm{mg} / \mathrm{L}$ atrazine with $14.34 \mathrm{mg} / \mathrm{L}$ atrazine-mancozeb mixture; $12.28 \mathrm{mg} / \mathrm{L}$ atrazine-chlorpyrifos mixture; $11.72 \mathrm{mg} / \mathrm{L}$ quaternary mixture; and $29.02 \mathrm{mg} / \mathrm{L}$ atrazine-lambda cyhalothrin mixture. $1 \mathrm{mg} / \mathrm{L}$ chlorpyrifos was compared to $0.57 \mathrm{mg} / \mathrm{L}$ chlorpyrifos-lambda cyhalothrin mixture, while $42 \mathrm{mg} / \mathrm{L}$ mancozeb with $7.74 \mathrm{mg} / \mathrm{L}$ mancozeb-chlorpyrifos, and $13.48 \mathrm{mg} / \mathrm{L}$ mancozeb-lambda cyhalothrin mixture. Our previous study provides details of the survival probabilities of the single pesticides used in this study for comparison with pesticide mixtures (Kanu et al., 2021a).

\section{Statistical analysis}

Student T-test was used to test the similarity of the weight, length, and condition factor of Nile tilapia and African catfish exposed to the pesticides. Student T-test was performed using SPSS version 22. The equality of survival distribution of fish exposed continuous and briefly for $15,30,45$, and $60 \mathrm{~min}$ to each pesticide was tested using the log-rank test. The Cox PH model 
was used to estimate the effect of species, pulse length, weight, fish length, and condition factor on fish survival. Survival analysis was performed using SAS version 9.1.3.

\section{Results}

Mean weight, length, and condition factor of fish species

Table 1 shows the average weight, length, and condition factor of fingerlings. The weight, length, and condition factor of African catfish fingerlings exposed to the pesticides were significantly different $(p<0.05)$ from Nile tilapia, except those indicated by the letter "a" were statistically similar $(p>0.05)$.

\section{Survival analysis \\ Effect on species difference on the survival of exposed fingerlings}

In Fig. 1a, with all covariates held constant, African catfish had a significantly higher $(p<0.05)$ survival probability than Nile tilapia after exposure to atrazinemancozeb mixture even though both species had similar (Table 1). On the other hand, Fig. 1b-g, show that the survival probability of African catfish exposed to atrazine-chlorpyrifos, atrazine-lambda cyhalothrin, mancozeb-chlorpyrifos, mancozeb-lambda cyhalothrin, chlorpyrifos-lambda cyhalothrin, and the quaternary mixture was similar to Nile tilapia fingerlings $(p>0.05)$. The survival probability of both species was identical despite the dissimilarity in weight of the fingerlings exposed to atrazine-lambda-cyhalothrin, mancozebchlorpyrifos, mancozeb-lambda cyhalothrin, and quaternary mixture (Table 1 ).

\section{Effect on exposure duration on the survival of exposed fingerlings}

Figure 2 shows the effect of exposure duration on the survival probability of African catfish and Nile tilapia fingerlings.

For both species, the survival probability was significantly lower for fingerlings continuously exposed to pesticide mixtures than those exposed by a pulse $(p<0.01)$. Nevertheless, the survival probability of tilapia exposed continuously to mancozeb-lambda cyhalothrin mixture was similar to $60 \mathrm{~min}$ pulse exposure $(p>0.05)$, suggesting the toxicity of $60 \mathrm{~min}$ pulse exposure was identical to continuous exposure for $96 \mathrm{~h}$.

Furthermore, the survival probability of Nile tilapia fingerlings exposed for 15, 30, 45 min to atrazine-chlorpyrifos, atrazine-lambda cyhalothrin, mancozeb-lambda cyhalothrin, and chlorpyrifos-lambda cyhalothrin differed significantly $(p<0.05)$ compared to $60 \mathrm{~min}$, indicating survival probability decreased as pulse duration increased.

\section{Effect of pesticide mixture on survival of fingerlings}

Figure 3 shows the survival graph of single fish exposed to single pesticides versus pesticide mixture. When the survival line of a pesticide mixture is significantly $(p<0.05)$ above that of a single pesticide in the graph, it indicates that fish survival probability was higher for the pesticide mixture than the single pesticide, which suggests an antagonistic interaction of the mixture components. On the other hand, when the survival line of a pesticide mixture is significantly $(p<0.05)$ below that of a single pesticide, it indicates the interaction of the mixture component was synergistic. Table 4 shows statistical estimates to buttress Fig. 3 .

\section{COX proportional hazard analysis \\ Effect on species difference on the survival of exposed fingerlings for different pulse exposure duration}

In Table 2, the survival probability of Nile tilapia decreased by a factor of 6.154, 2.639, 3.282, and 2.703 compared with African catfish fingerlings exposed to atrazine-chlorpyrifos, atrazine-lambda cyhalothrin, and mancozeb-lambda cyhalothrin for $60 \mathrm{~min}$ and chlorpyrifos-lambda cyhalothrin for $45 \mathrm{~min}$, respectively. The survival probability of both species was similar after 15,30 , and 45 min of pulse exposure $(p>0.05)$.

\section{Effect of pulse duration on the hazard of pesticide mixture}

In Table 3, the survival probability of African catfish exposed to atrazine-mancozeb, atrazine-chlorpyrifos, atrazine-lambda cyhalothrin, mancozeb-chlorpyrifos, mancozeb-lambda cyhalothrin, and Nile tilapia exposed to atrazine-mancozeb, mancozeb-chlorpyrifos, and quaternary mixture for $15,30,45 \mathrm{~min}$, and $60 \mathrm{~min}$ was not significantly different $(p>0.05)$. However, the survival probability of Nile tilapia fingerlings exposed to atrazinechlorpyrifos for 15, 30, 45 min was 0.145 (86\%), 0.199 (80\%), and 0.289 (71\%) higher than those exposed for $60 \mathrm{~min}$, respectively. In addition, Nile tilapia exposed to atrazine-lambda cyhalothrin for 15 and 30 min were $0.102(90 \%)$ and $0.210(79 \%)$ times less likely to die than those exposed for $60 \mathrm{~min}$. Tilapia fingerlings exposed to mancozeb-Lambda cyhalothrin 15, 30, 45 min were 0.099 (90\%), $0.212(79 \%)$, and $0.323(68 \%)$ times less likely to die compared to those exposed for $60 \mathrm{~min}$. African catfish exposed to chlorpyrifos-lambda cyhalothrin and quaternary mixture for $15 \mathrm{~min}$ were $0.319(68 \%)$ and $0.336(66 \%)$ times less likely to die, respectively, relative to those exposed for $60 \mathrm{~min}$. In contrast, the probability of survival of Nile tilapia exposed to chlorpyrifos-lambda cyhalothrin for 15 min was higher by 0.117 (88\%) than those exposed for $60 \mathrm{~min}$. 
Table 1 Fingerlings mean weight, length, and condition factor

\begin{tabular}{|c|c|c|c|c|c|c|c|c|c|}
\hline \multirow{2}{*}{$\begin{array}{l}\text { Exposure } \\
\text { duration }\end{array}$} & \multirow[t]{2}{*}{ Variables } & \multirow[t]{2}{*}{ Fish } & \multicolumn{7}{|c|}{ Pesticide mixture } \\
\hline & & & $\begin{array}{l}\text { Atrazine: } \\
\text { Mancozeb }\end{array}$ & $\begin{array}{l}\text { Atrazine: } \\
\text { Chlorpyrifos }\end{array}$ & $\begin{array}{l}\text { Atrazine: } \\
\text { Lambda } \\
\text { cyhalothrin }\end{array}$ & $\begin{array}{l}\text { Mancozeb: } \\
\text { Chlorpyrifos }\end{array}$ & $\begin{array}{l}\text { Mancozeb: } \\
\text { Lambda } \\
\text { cyhalothrin }\end{array}$ & $\begin{array}{l}\text { Lambda } \\
\text { cyhalothrin: } \\
\text { Chlorpyrifos }\end{array}$ & Quaternary \\
\hline \multirow[t]{6}{*}{ Continuous } & Weight & $\begin{array}{l}\text { African } \\
\text { catfish }\end{array}$ & $0.48 \pm 0.03^{\mathrm{a}}$ & $0.4 \pm 0.02^{\mathrm{a}}$ & $0.44 \pm 0.02$ & $0.44 \pm 0.03$ & $0.41 \pm 0.03$ & $0.42 \pm 0.02^{\mathrm{a}}$ & $0.55 \pm 0.03$ \\
\hline & & Tilapia & $0.43 \pm 0.1^{\mathrm{a}}$ & $0.53 \pm 0.11^{\mathrm{a}}$ & $0.23 \pm 0.02$ & $0.83 \pm 0.08$ & $1.44 \pm 0.21$ & $0.57 \pm 0.09^{a}$ & $0.4 \pm 0.05$ \\
\hline & Length & $\begin{array}{l}\text { African } \\
\text { catfish }\end{array}$ & $3.91 \pm 0.07$ & $3.99 \pm 0.07$ & $3.92 \pm 0.08$ & $3.8 \pm 0.09$ & $3.73 \pm 0.05^{\mathrm{a}}$ & $3.6 \pm 0.07$ & $4.06 \pm 0.09$ \\
\hline & & Tilapia & $2.71 \pm 0.16$ & $3.01 \pm 0.2$ & $2.52 \pm 0.14$ & $3.23 \pm 0.15$ & $3.94 \pm 0.24^{\mathrm{a}}$ & $2.98 \pm 0.18$ & $2.8 \pm 0.15$ \\
\hline & $\begin{array}{l}\text { Condition } \\
\text { Factor }\end{array}$ & $\begin{array}{l}\text { African } \\
\text { catfish }\end{array}$ & $0.8 \pm 0.05$ & $0.63 \pm 0.03$ & $0.76 \pm 0.04$ & $0.81 \pm 0.06$ & $0.79 \pm 0.06$ & $0.96 \pm 0.07$ & $0.83 \pm 0.04$ \\
\hline & & Tilapia & $1.95 \pm 0.12$ & $1.64 \pm 0.14$ & $1.61 \pm 0.16$ & $2.34 \pm 0.09$ & $1.98 \pm 0.05$ & $1.98 \pm 0.11$ & $1.82 \pm 0.12$ \\
\hline \multirow[t]{6}{*}{15 Min } & Weight & $\begin{array}{l}\text { African } \\
\text { catfish }\end{array}$ & $0.57 \pm 0.01^{a}$ & $0.62 \pm 0.01$ & $0.42 \pm 0.01$ & $0.44 \pm 0.08^{\mathrm{a}}$ & $0.38 \pm 0.02$ & $0.31 \pm 0.01^{\mathrm{a}}$ & $0.52 \pm 0.03^{a}$ \\
\hline & & Tilapia & $0.52 \pm 0.09^{\mathrm{a}}$ & $0.38 \pm 0.04$ & $0.27 \pm 0.02$ & $0.41 \pm 0.04^{\mathrm{a}}$ & $0.29 \pm 0.03$ & $0.27 \pm 0.02^{\mathrm{a}}$ & $0.55 \pm 0.05^{\mathrm{a}}$ \\
\hline & Length & $\begin{array}{l}\text { African } \\
\text { catfish }\end{array}$ & $4.38 \pm 0.12$ & $4.34 \pm 0.06$ & $3.88 \pm 0.11$ & $3.85 \pm 0.1^{\mathrm{a}}$ & $3.7 \pm 0.09$ & $3.19 \pm 0.08$ & $4.34 \pm 0.05$ \\
\hline & & Tilapia & $2.95 \pm 0.18$ & $2.85 \pm 0.08$ & $2.47 \pm 0.06$ & $3.58 \pm 0.27^{\mathrm{a}}$ & $2.43 \pm 0.1$ & $2.51 \pm 0.09$ & $4 \pm 0.1$ \\
\hline & $\begin{array}{l}\text { Condition } \\
\text { Factor }\end{array}$ & $\begin{array}{l}\text { African } \\
\text { catfish }\end{array}$ & $0.72 \pm 0.05$ & $0.78 \pm 0.05$ & $0.77 \pm 0.06$ & $0.78 \pm 0.13^{\mathrm{a}}$ & $0.75 \pm 0.04$ & $0.96 \pm 0.05$ & $0.65 \pm 0.03$ \\
\hline & & Tilapia & $2.07 \pm 0.15$ & $1.62 \pm 0.08$ & $1.85 \pm 0.12$ & $1.23 \pm 0.2^{\mathrm{a}}$ & $2.04 \pm 0.12$ & $1.73 \pm 0.13$ & $0.91 \pm 0.09$ \\
\hline \multirow[t]{6}{*}{30 Min } & Weight & $\begin{array}{l}\text { African } \\
\text { catfish }\end{array}$ & $0.52 \pm 0.01$ & $0.88 \pm 0.05$ & $0.43 \pm 0.0^{\mathrm{a}}$ & $0.36 \pm 0.01^{\mathrm{a}}$ & $0.5 \pm 0.03$ & $0.35 \pm 0.02$ & $0.72 \pm 0.07$ \\
\hline & & Tilapia & $0.33 \pm 0.03$ & $0.43 \pm 0.04$ & $0.4 \pm 0.06^{\mathrm{a}}$ & $0.42 \pm 0.05^{\mathrm{a}}$ & $0.8 \pm 0.1$ & $0.61 \pm 0.16$ & $1.07 \pm 0.14$ \\
\hline & Length & $\begin{array}{l}\text { African } \\
\text { catfish }\end{array}$ & $4.04 \pm 0.08$ & $4.88 \pm 0.14$ & $3.74 \pm 0.08$ & $3.54 \pm 0.05^{\mathrm{a}}$ & $4.08 \pm 0.11$ & $3.38 \pm 0.08$ & $5.72 \pm 0.26$ \\
\hline & & Tilapia & $2.48 \pm 0.07$ & $2.99 \pm 0.12$ & $2.74 \pm 0.14$ & $3.71 \pm 0.28^{\mathrm{a}}$ & $3.52 \pm 0.19$ & $2.97 \pm 0.18$ & $3.89 \pm 0.2$ \\
\hline & $\begin{array}{l}\text { Condition } \\
\text { Factor }\end{array}$ & $\begin{array}{l}\text { African } \\
\text { catfish }\end{array}$ & $0.8 \pm 0.05$ & $0.79 \pm 0.06$ & $0.87 \pm 0.05$ & $0.81 \pm 0.02^{\mathrm{a}}$ & $0.75 \pm 0.04$ & $0.9 \pm 0.04$ & $0.39 \pm 0.03$ \\
\hline & & Tilapia & $2.23 \pm 0.17$ & $1.57 \pm 0.05$ & $2.05 \pm 0.28$ & $1.06 \pm 0.15^{\mathrm{a}}$ & $1.75 \pm 0.1$ & $1.91 \pm 0.12$ & $1.7 \pm 0.1$ \\
\hline \multirow[t]{6}{*}{45 Min } & Weight & $\begin{array}{l}\text { African } \\
\text { catfish }\end{array}$ & $0.47 \pm 0.01^{a}$ & $0.84 \pm 0.04$ & $0.44 \pm 0.01^{\mathrm{a}}$ & $0.39 \pm 0.02$ & $0.52 \pm 0.03^{\mathrm{a}}$ & $0.35 \pm 0.02$ & $0.59 \pm 0.05$ \\
\hline & & Tilapia & $0.59 \pm 0.07^{a}$ & $0.44 \pm 0.05$ & $0.42 \pm 0.08^{\mathrm{a}}$ & $0.96 \pm 0.17$ & $0.47 \pm 0.05^{\mathrm{a}}$ & $0.96 \pm 0.1$ & $0.44 \pm 0.03$ \\
\hline & Length & $\begin{array}{l}\text { African } \\
\text { catfish }\end{array}$ & $3.93 \pm 0.08$ & $4.81 \pm 0.12$ & $3.9 \pm 0.09$ & $3.85 \pm 0.1^{\mathrm{a}}$ & $4.06 \pm 0.14$ & $3.47 \pm 0.07^{a}$ & $4.93 \pm 0.16$ \\
\hline & & Tilapia & $3.13 \pm 0.17$ & $2.99 \pm 0.15$ & $2.68 \pm 0.18$ & $4.03 \pm 0.32^{\mathrm{a}}$ & $2.98 \pm 0.15$ & $3.53 \pm 0.16^{a}$ & $3.3 \pm 0.13$ \\
\hline & $\begin{array}{l}\text { Condition } \\
\text { Factor }\end{array}$ & $\begin{array}{l}\text { African } \\
\text { catfish }\end{array}$ & $0.81 \pm 0.05$ & $0.79 \pm 0.04$ & $0.77 \pm 0.05$ & $0.68 \pm 0.02$ & $0.82 \pm 0.06$ & $0.84 \pm 0.03$ & $0.49 \pm 0.03$ \\
\hline & & Tilapia & $1.94 \pm 0.15$ & $1.64 \pm 0.09$ & $1.95 \pm 0.1$ & $1.72 \pm 0.32$ & $1.7 \pm 0.08$ & $2.01 \pm 0.08$ & $1.28 \pm 0.11$ \\
\hline \multirow[t]{6}{*}{$60 \mathrm{Min}$} & Weight & $\begin{array}{l}\text { African } \\
\text { catfish }\end{array}$ & $0.53 \pm 0.01$ & $0.75 \pm 0.05$ & $0.44 \pm 0.01^{\mathrm{a}}$ & $0.37 \pm 0.02$ & $0.61 \pm 0.01$ & $0.37 \pm 0.02^{\mathrm{a}}$ & $0.6 \pm 0.06^{a}$ \\
\hline & & Tilapia & $0.41 \pm 0.05$ & $0.51 \pm 0.07$ & $0.4 \pm 0.05^{\mathrm{a}}$ & $0.75 \pm 0.17$ & $0.96 \pm 0.18$ & $0.43 \pm 0.08^{a}$ & $0.57 \pm 0.05^{a}$ \\
\hline & Length & $\begin{array}{l}\text { African } \\
\text { catfish }\end{array}$ & $4.21 \pm 0.07$ & $4.6 \pm 0.15$ & $3.69 \pm 0.07$ & $3.59 \pm 0.08^{\mathrm{a}}$ & $4.44 \pm 0.12$ & $3.56 \pm 0.09$ & $5 \pm 0.22$ \\
\hline & & Tilapia & $2.79 \pm 0.19$ & $2.94 \pm 0.13$ & $2.92 \pm 0.18$ & $3.31 \pm 0.25^{\mathrm{a}}$ & $3.28 \pm 0.24$ & $2.86 \pm 0.17$ & $3.43 \pm 0.18$ \\
\hline & $\begin{array}{l}\text { Condition } \\
\text { Factor }\end{array}$ & $\begin{array}{l}\text { African } \\
\text { catfish }\end{array}$ & $0.72 \pm 0.03$ & $0.83 \pm 0.08$ & $0.91 \pm 0.05$ & $0.81 \pm 0.03$ & $0.74 \pm 0.05$ & $0.8 \pm 0.03$ & $0.5 \pm 0.03$ \\
\hline & & Tilapia & $2.07 \pm 0.17$ & $2.06 \pm 0.41$ & $1.82 \pm 0.28$ & $1.76 \pm 0.15$ & $2.28 \pm 0.08$ & $1.69 \pm 0.12$ & $1.55 \pm 0.16$ \\
\hline
\end{tabular}




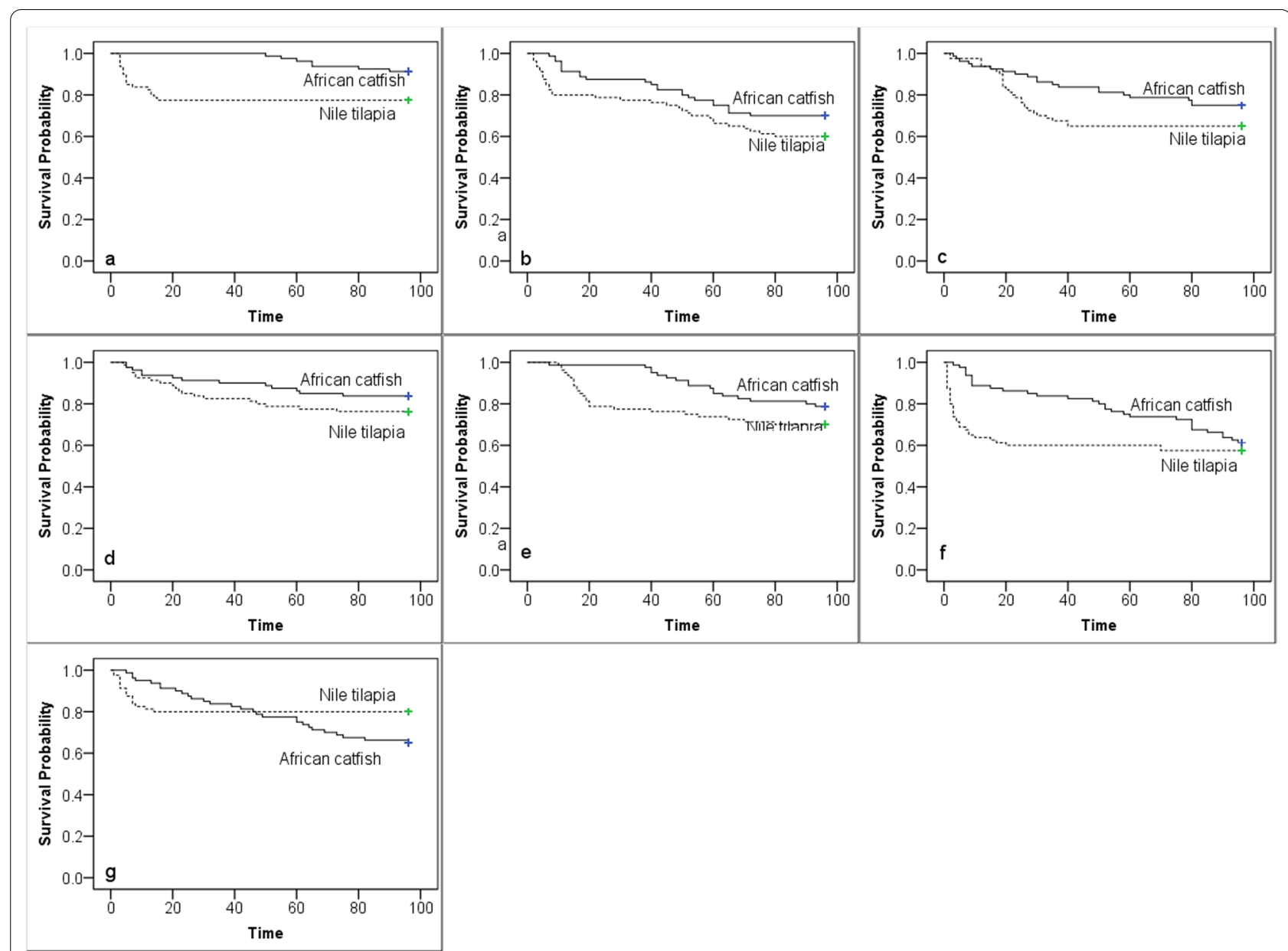

Fig. 1 Survival probability of Fingerings exposed to pesticide mixtures. $\mathbf{a}, \mathbf{b}, \mathbf{c}, \mathbf{d}, \mathbf{e}, \mathbf{f}, \mathbf{g}$ Atrazine-mancozeb, atrazine-chlorpyrifos, atrazine-lambda cyhalothrin, mancozeb-chlorpyrifos, mancozeb-lambda cyhalothrin, chlorpyrifos-lambda cyhalothrin, and quaternary mixture

\section{Pesticide mixture effect on survival of fingerlings}

Table 4 shows that overall, the survival probability of Nile tilapia fingerlings pulse exposed to atrazine was not significantly different $(p>0.05)$ from those exposed to atrazine-mancozeb, atrazine-chlorpyrifos, atrazinelambda cyhalothrin, and the quaternary pesticide mixture. Similarly, Nile tilapia fingerlings pulsed exposed to mancozeb was not significantly different $(p>0.05)$ from those exposed to mancozeb-chlorpyrifos and mancozeb-lambda cyhalothrin mixture, which suggests that pulse exposure to the pesticide mixture containing binary or quaternary active ingredients was not more toxic to tilapia than pesticides with one active ingredient. It also indicates no interaction of mixture components. However, survival probability was significantly higher $(p<0.05)$ for Nile tilapia fingerlings' pulse exposed to chlorpyrifos-lambda cyhalothrin than chlorpyrifos, which indicates that the mixture was less toxic than the single pesticide, suggesting an antagonistic interaction between chlorpyrifos and lambda-cyhalothrin tilapia fingerlings. The reverse occurred for African catfish fish fingerlings.

The survival probability of African catfish fingerling's pulse exposed to atrazine was significantly lower $(p<0.05)$ than atrazine-mancozeb, atrazine-chlorpyrifos, atrazine-lambda cyhalothrin, and the quaternary pesticide mixture by a factor of $7.83,1.88,2.29$, and 1.59 , respectively. Likewise, fingerlings pulsed exposed to mancozeb was significantly lower $(p<0.05)$ than fingerlings exposed to mancozeb-chlorpyrifos and mancozeb-lambda cyhalothrin mixture by a factor of 3.15 and 2.51, respectively. Lower pesticide mixture toxicity suggests an antagonistic effect of the pesticide mixture in African catfish. Nevertheless, survival probability was not significantly different $(p>0.05)$ for African catfish fingerling's pulse exposed to chlorpyrifos-lambda cyhalothrin compared with chlorpyrifos. 


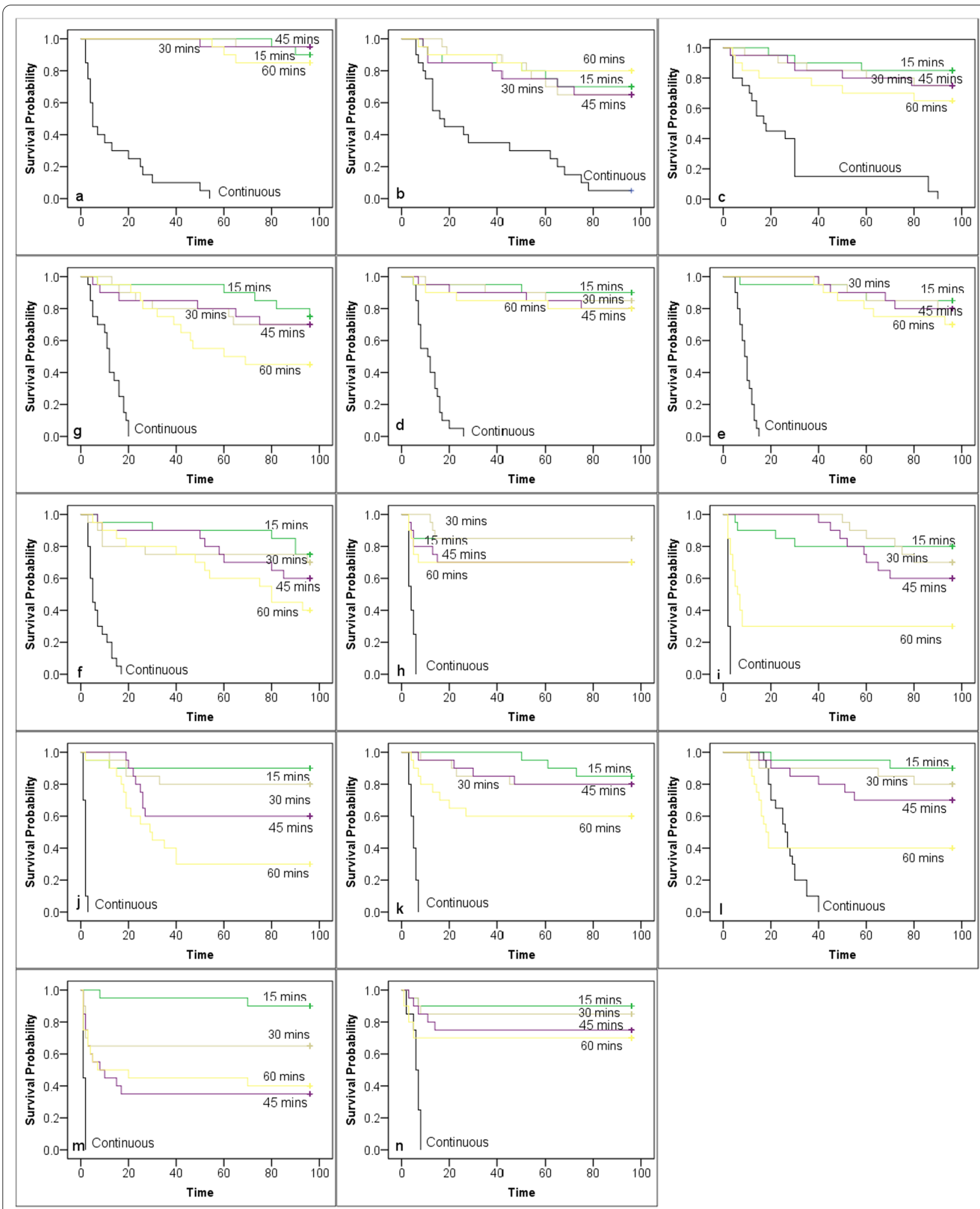

Fig. 2 Influence of pulse duration on African catfish and Nile tilapia survival. Letters $\mathbf{a}, \mathbf{b}, \mathbf{c}, \mathbf{d}, \mathbf{e}, \mathbf{f}, \mathbf{g}$-African catfish exposed to atrazine-mancozeb, atrazine-chlorpyrifos, atrazine-lambda cyhalothrin, mancozeb-chlorpyrifos, mancozeb-lambda cyhalothrin, chlorpyrifos-lambda cyhalothrin, and quaternary mixture, respectively, while $\mathbf{h}, \mathbf{i}, \mathbf{j}, \mathbf{k}, \mathbf{l}, \mathbf{m}, \mathbf{n}$-Nile tilapia exposed to atrazine-mancozeb, atrazine-chlorpyrifos, atrazine-lambda cyhalothrin, mancozeb-chlorpyrifos, mancozeb-lambda cyhalothrin, chlorpyrifos-lambda cyhalothrin, and quaternary mixture, respectively 

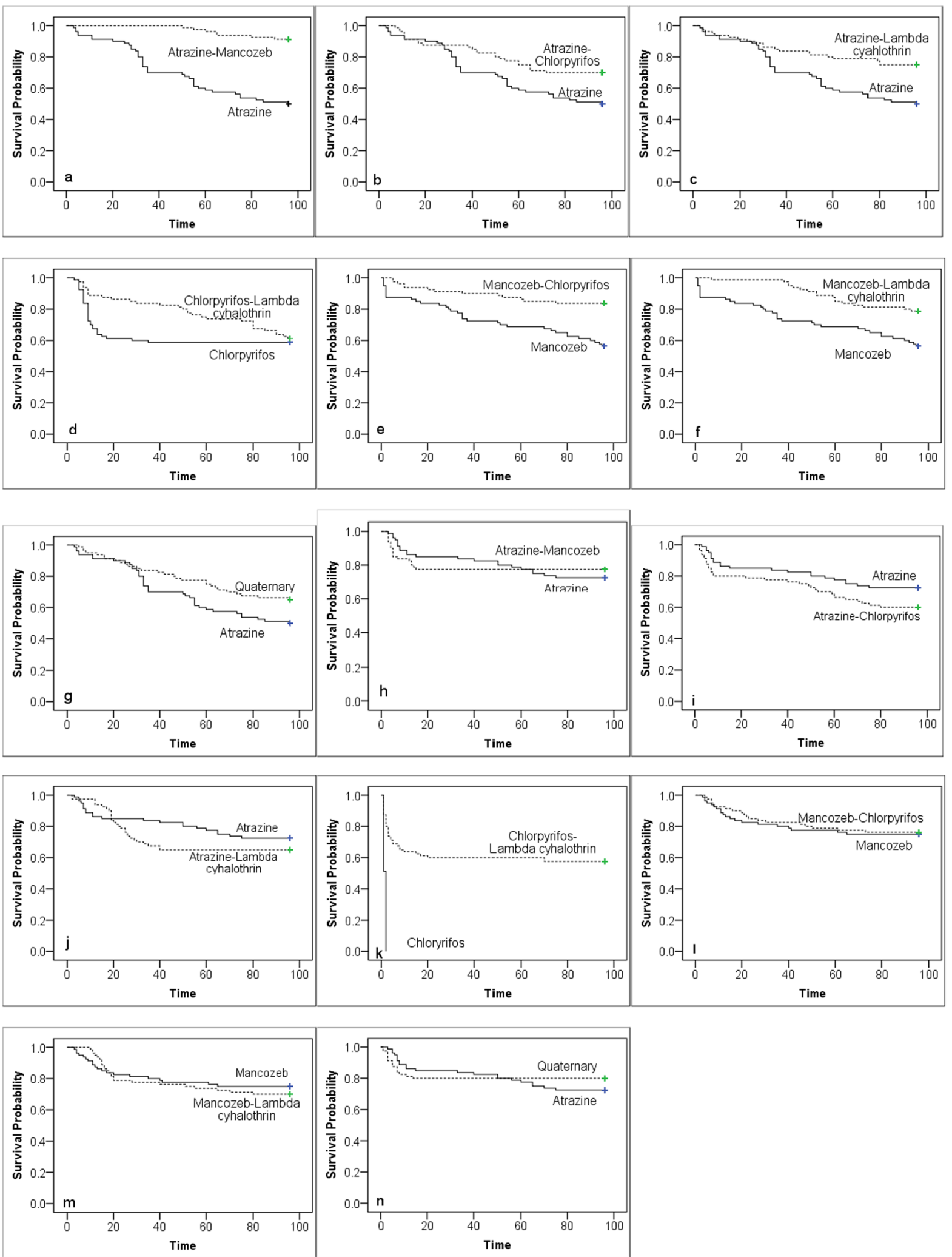

Fig. 3 Survival graph of fish exposed to single pesticides versus pesticide mixture. Letters $\mathbf{a}, \mathbf{b}, \mathbf{c}, \mathbf{d}, \mathbf{e}, \mathbf{f}$, and $g$ are survival graphs of African catfish, $\mathbf{h}, \mathbf{i}, \mathbf{j}, \mathbf{k}, \mathbf{l}, \mathbf{m}$ and $\mathbf{n}$ are survival graphs for tilapia 
Table 2 Cox PH model summary of species effect on survival of fingerlings at different pulse exposure duration

\begin{tabular}{|c|c|c|c|c|c|c|c|}
\hline Pesticide & $\begin{array}{l}\text { Exposure } \\
\text { duration (mins) }\end{array}$ & $d f$ & $\begin{array}{l}\text { Parameter } \\
\text { estimate }\end{array}$ & SE & Chi-square & $\operatorname{Pr}>$ ChiSq & Hazard ratio \\
\hline \multirow[t]{4}{*}{ Atrazine-Mancozeb } & $15^{*}$ & 1 & .482 & .913 & .278 & .598 & 1.619 \\
\hline & $30^{*}$ & 1 & 1.179 & 1.155 & 1.042 & .307 & 3.252 \\
\hline & $45^{*}$ & 1 & 1.958 & 1.081 & 3.281 & .070 & 7.088 \\
\hline & $60^{*}$ & 1 & .877 & .708 & 1.533 & .216 & 2.404 \\
\hline \multirow[t]{4}{*}{ Atrazine-Chlorpyrifos } & $15^{*}$ & 1 & -.391 & 646 & .366 & .545 & .677 \\
\hline & $30^{*}$ & 1 & -.300 & .557 & .291 & .590 & .741 \\
\hline & $45^{*}$ & 1 & .074 & .518 & .020 & .887 & 1.077 \\
\hline & $60^{*}$ & 1 & 1.817 & .574 & 10.027 & .002 & 6.154 \\
\hline \multirow[t]{4}{*}{ Atrazine-Lambda cyhalothrin } & $15^{*}$ & 1 & -.363 & .913 & .158 & .691 & .695 \\
\hline & $30^{*}$ & 1 & -.190 & .671 & .081 & .777 & .827 \\
\hline & $45^{*}$ & 1 & .670 & .572 & 1.372 & .241 & 1.954 \\
\hline & $60^{*}$ & 1 & .970 & .469 & 4.285 & .038 & 2.639 \\
\hline \multirow[t]{4}{*}{ Mancozeb-Chlorpyrifos } & $15^{*}$ & 1 & .374 & .913 & .168 & .682 & 1.454 \\
\hline & $30^{*}$ & 1 & .344 & .764 & .202 & .653 & 1.410 \\
\hline & $45^{*}$ & 1 & .023 & .707 & .001 & .974 & 1.023 \\
\hline & $60^{*}$ & 1 & .856 & .613 & 1.950 & .163 & 2.354 \\
\hline \multirow[t]{4}{*}{ Mancozeb-Lambda cyhalothrin } & $15^{*}$ & 1 & -.449 & .913 & .242 & .623 & .638 \\
\hline & $30^{*}$ & 1 & .027 & .707 & .001 & .970 & 1.027 \\
\hline & $45^{*}$ & 1 & .546 & .646 & .714 & .398 & 1.726 \\
\hline & $60^{*}$ & 1 & 1.188 & .505 & 5.547 & .019 & 3.282 \\
\hline \multirow[t]{4}{*}{ Chlorpyrifos-Lambda cyhalothrin } & $15^{*}$ & 1 & -.948 & .837 & 1.283 & .257 & .388 \\
\hline & $30^{*}$ & 1 & .348 & .558 & .390 & .532 & 1.417 \\
\hline & $45^{*}$ & 1 & .994 & .454 & 4.800 & .028 & 2.703 \\
\hline & $60^{*}$ & 1 & .340 & .410 & .686 & .408 & 1.405 \\
\hline \multirow[t]{4}{*}{ Quaternary } & $15^{*}$ & 1 & -.920 & .837 & 1.207 & .272 & .399 \\
\hline & $30^{*}$ & 1 & -.661 & .708 & .872 & .350 & .516 \\
\hline & $45^{*}$ & 1 & -.108 & .606 & .032 & .858 & .897 \\
\hline & $60^{*}$ & 1 & -.543 & .510 & 1.134 & .287 & .581 \\
\hline
\end{tabular}

"Hazard ratio constant with time; $\mathrm{Pr}>\mathrm{ChiSq}$ indicates statistical significance if $<0.05$

The combined effect of species, pulse duration, weight, length, and condition factor on the survival of fingerlings

Table 5, taking all predictors into account, pulse toxicity of atrazine-mancozeb was not significantly associated with any covariate $(p>0.05)$. The survival probability of Nile tilapia and African catfish fingerling exposed to $14.34 \mathrm{mg} / \mathrm{L}$ atrazine-mancozeb mixture was similar. Pulse length, fish weight \& length, and condition factor did not influence the survival of both species even though African catfish's fish length and condition factor were significantly longer and smaller, respectively, than Nile tilapia fingerlings (Table 1).

Pulse toxicity of $12.58 \mathrm{mg} / \mathrm{L}$ mancozeb-chlorpyrifos was associated with $15 \mathrm{~min}$ pulse length and fish weight $(p<0.05)$. The likelihood of survival after $15 \mathrm{~min}$ exposure increased by a factor of 0.312 compared to $60 \mathrm{~min}$ $(p<0.05)$, while survival likelihood increased by $99.1 \%$ in fishes weighing $0.1 \mathrm{~g}$ more than other fish. The toxicity of pulse exposure to $12.58 \mathrm{mg} / \mathrm{L}$ for 30 and $45 \mathrm{~min}$ was similar to $60 \mathrm{~min}$ irrespective of the species, length, and condition factor difference (Table 1).

Pulse toxicity of $29.02 \mathrm{mg} / \mathrm{L}$ atrazine-lambda cyhalothrin was associated with pulse length $(p<0.05)$ alone. The likelihood of survival after 15,30 , and 45 min exposure increased by a factor of $0.120(88 \%), 0.275(72.5 \%)$, $0.380(62 \%)$, respectively, compared to $60 \mathrm{~min}$ pulse exposure $(p<0.05)$ irrespective of the difference in specie, weight, length and condition factor (Table 1).

Pulse toxicity of $7.74 \mathrm{mg} / \mathrm{L}$ mancozeb-chlorpyrifos was associated with 15 pulse length and fish weight $(p<0.05)$. The likelihood of survival after $15 \mathrm{~min}$ of exposure increased by a factor of 0.313 compared to 60 $(p<0.05)$. The toxicity of pulse exposure to $7.74 \mathrm{mg} / \mathrm{L}$ mancozeb-chlorpyrifos for 30 and $45 \mathrm{~min}$ was similar to $60 \mathrm{~min}$ pulse exposure $(p<0.05)$ irrespective of the difference in species, weight, length, and condition factor (Table 1). 
Table 3 Cox PH model summary of the effect of pulse duration on the hazard of pesticide mixture

\begin{tabular}{|c|c|c|c|c|c|c|c|}
\hline Pesticide & Species & & $d f$ & Parameter estimate & SE & Chi-square & Hazard ratio \\
\hline \multirow[t]{8}{*}{ Atrazine-Mancozeb } & African catfish* & $15 \mathrm{~min}$ & 1 & $-.482^{b}$ & .913 & .279 & $.618^{\mathrm{b}}$ \\
\hline & & $30 \mathrm{~min}$ & 1 & $-1.168^{b}$ & 1.155 & 1.022 & $.311^{\mathrm{b}}$ \\
\hline & & $45 \mathrm{~min}$ & 1 & $-1.138^{b}$ & 1.155 & .972 & $.320^{\mathrm{b}}$ \\
\hline & & $60 \mathrm{~min}$ & 3 & & & 1.596 & \\
\hline & Tilapia* & $15 \mathrm{~min}$ & 1 & $-.754^{\mathrm{b}}$ & .707 & 1.137 & $.470^{\mathrm{b}}$ \\
\hline & & $30 \mathrm{~min}$ & 1 & $-.843^{b}$ & .708 & 1.418 & $.431^{\mathrm{b}}$ \\
\hline & & $45 \mathrm{~min}$ & 1 & $-.054^{\mathrm{b}}$ & .577 & .009 & $.947^{b}$ \\
\hline & & $60 \mathrm{~min}$ & 3 & & & 2.397 & \\
\hline \multirow[t]{8}{*}{ Atrazine-Chlorpyrifos } & African catfish* & $15 \min$ & 1 & $.423^{b}$ & .646 & .429 & $1.526^{\mathrm{b}}$ \\
\hline & & $30 \mathrm{~min}$ & 1 & $.559^{\mathrm{b}}$ & .627 & .795 & $1.749^{b}$ \\
\hline & & $45 \mathrm{~min}$ & 1 & $.610^{\mathrm{b}}$ & .627 & .947 & $1.840^{\mathrm{b}}$ \\
\hline & & $60 \mathrm{~min}$ & 3 & & & 1.076 & \\
\hline & Tilapia & $15 \mathrm{~min}$ & 1 & $-1.931^{\mathrm{a}}$ & .571 & 11.455 & $.145^{\mathrm{a}}$ \\
\hline & & $30 \mathrm{~min}$ & 1 & $-1.612^{\mathrm{a}}$ & .493 & 10.675 & $.199^{\mathrm{a}}$ \\
\hline & & $45 \mathrm{~min}$ & 1 & $-1.242^{\mathrm{a}}$ & .448 & 7.679 & $.289^{\mathrm{a}}$ \\
\hline & & $60 \mathrm{~min}$ & 3 & & & 18.875 & \\
\hline \multirow[t]{8}{*}{ Atrazine-Lambda cyhalothrin } & African catfish* & $15 \min$ & 1 & $-.998^{\mathrm{b}}$ & .690 & 2.092 & $.369^{b}$ \\
\hline & & $30 \mathrm{~min}$ & 1 & $-.453^{\mathrm{b}}$ & .586 & .599 & $.636^{\mathrm{b}}$ \\
\hline & & $45 \mathrm{~min}$ & 1 & $-.431^{\mathrm{b}}$ & .586 & .540 & $.650^{\mathrm{b}}$ \\
\hline & & $60 \mathrm{~min}$ & 3 & & & 2.194 & \\
\hline & Tilapia & $15 \mathrm{~min}$ & 1 & $-2.279^{a}$ & .758 & 9.054 & $.102^{\mathrm{a}}$ \\
\hline & & $30 \mathrm{~min}$ & 1 & $-1.561^{\mathrm{a}}$ & .568 & 7.550 & $.210^{\mathrm{a}}$ \\
\hline & & $45 \mathrm{~min}$ & 1 & $-.793^{b}$ & .444 & 3.196 & $.452^{\mathrm{b}}$ \\
\hline & & $60 \mathrm{~min}$ & 3 & & & 14.563 & \\
\hline \multirow[t]{8}{*}{ Mancozeb-Chlorpyrifos } & African catfish* & $15 \min$ & 1 & $-.741^{\mathrm{b}}$ & .866 & .732 & $.477^{\mathrm{b}}$ \\
\hline & & $30 \mathrm{~min}$ & 1 & $-.334^{\mathrm{b}}$ & .764 & .191 & $.716^{\mathrm{b}}$ \\
\hline & & $45 \mathrm{~min}$ & 1 & $-.026^{\mathrm{b}}$ & .707 & .001 & $.974^{\mathrm{b}}$ \\
\hline & & $60 \mathrm{~min}$ & 3 & & & .921 & \\
\hline & Tilapia & $15 \mathrm{~min}$ & 1 & $-1.303^{b}$ & .678 & 3.694 & $.272^{\mathrm{b}}$ \\
\hline & & $30 \mathrm{~min}$ & 1 & $-.925^{\mathrm{b}}$ & .613 & 2.279 & $.396^{\mathrm{b}}$ \\
\hline & & $45 \mathrm{~min}$ & 1 & $-.932^{\mathrm{b}}$ & .613 & 2.314 & $.394^{b}$ \\
\hline & & $60 \mathrm{~min}$ & 3 & & & 5.206 & \\
\hline \multirow[t]{8}{*}{ Mancozeb-Lambda cyhalothrin } & African catfish* & $15 \min$ & 1 & $-.760^{\mathrm{b}}$ & .707 & 1.154 & $.468^{b}$ \\
\hline & & $30 \mathrm{~min}$ & 1 & $-.477^{\mathrm{b}}$ & .646 & .547 & $.620^{\mathrm{b}}$ \\
\hline & & $45 \mathrm{~min}$ & 1 & $-.471^{\mathrm{b}}$ & .646 & .533 & $.624^{b}$ \\
\hline & & $60 \mathrm{~min}$ & 3 & & & 1.361 & \\
\hline & Tilapia & $15 \min$ & 1 & $-2.315^{\mathrm{a}}$ & .767 & 9.116 & $.099^{\mathrm{a}}$ \\
\hline & & $30 \mathrm{~min}$ & 1 & $-1.549^{\mathrm{a}}$ & .581 & 7.111 & $.212^{\mathrm{a}}$ \\
\hline & & $45 \mathrm{~min}$ & 1 & $-1.131^{\mathrm{a}}$ & .503 & 5.051 & $.323^{\mathrm{a}}$ \\
\hline & & $60 \mathrm{~min}$ & 3 & & & 14.770 & \\
\hline \multirow[t]{8}{*}{ Chlorpyrifos-Lambda cyhalothrin } & African catfish* & $15 \mathrm{~min}$ & 1 & $-1.144^{\mathrm{a}}$ & .534 & 4.591 & $.319^{\mathrm{a}}$ \\
\hline & & $30 \mathrm{~min}$ & 1 & $-.834^{b}$ & .502 & 2.768 & $.434^{\mathrm{b}}$ \\
\hline & & $45 \mathrm{~min}$ & 1 & $-.557^{b}$ & .457 & 1.487 & $.573^{b}$ \\
\hline & & $60 \mathrm{~min}$ & 3 & & & 5.718 & \\
\hline & Tilapia* & $15 \mathrm{~min}$ & 1 & $-2.146^{\mathrm{a}}$ & .765 & 7.868 & $.117^{\mathrm{a}}$ \\
\hline & & $30 \mathrm{~min}$ & 1 & $-.644^{\mathrm{b}}$ & .476 & 1.828 & $.525^{b}$ \\
\hline & & $45 \mathrm{~min}$ & 1 & $.084^{\mathrm{b}}$ & .401 & .044 & $1.087^{b}$ \\
\hline & & $60 \mathrm{~min}$ & 3 & & & 10.384 & \\
\hline
\end{tabular}


Table 3 (continued)

\begin{tabular}{|c|c|c|c|c|c|c|c|}
\hline Pesticide & Species & & $d f$ & Parameter estimate & SE & Chi-square & Hazard ratio \\
\hline \multirow[t]{8}{*}{ Quaternary } & African catfish* & $15 \mathrm{~min}$ & 1 & $-1.090^{\mathrm{a}}$ & .542 & 4.051 & $.336^{\mathrm{a}}$ \\
\hline & & $30 \mathrm{~min}$ & 1 & $-.784^{b}$ & .509 & 2.374 & $.457^{\mathrm{b}}$ \\
\hline & & $45 \mathrm{~min}$ & 1 & $-.797^{\mathrm{b}}$ & .509 & 2.446 & $.451^{\mathrm{b}}$ \\
\hline & & $60 \mathrm{~min}$ & 3 & & & 5.407 & \\
\hline & Tilapia* & $15 \mathrm{~min}$ & 1 & $-1.268^{b}$ & .817 & 2.409 & $.281^{\mathrm{b}}$ \\
\hline & & $30 \mathrm{~min}$ & 1 & $-.855^{b}$ & .707 & 1.460 & $.425^{\mathrm{b}}$ \\
\hline & & $45 \mathrm{~min}$ & 1 & $-.326^{\mathrm{b}}$ & .606 & .290 & $.722^{\mathrm{b}}$ \\
\hline & & $60 \mathrm{~min}$ & 3 & & & 3.104 & \\
\hline
\end{tabular}

*Hazard ratio constant with time; a- indicates statistical significance $(p<0.05)$; b- indicates not significant $(p>0.05)$

Table 4 Cox PH model summary of pesticide mixture effect on survival of fingerlings

\begin{tabular}{|c|c|c|c|c|c|c|c|}
\hline Fish species & Compared pesticides & $d f$ & $\begin{array}{l}\text { Parameter } \\
\text { estimate }\end{array}$ & SE & Chi-square & $\operatorname{Pr}>$ ChiSq & Hazard ratio \\
\hline \multirow[t]{7}{*}{ African catfish } & Atrazine vs. Atrazine-Mancozeb & 1 & 2.059 & .411 & 25.142 & .000 & 7.834 \\
\hline & Mancozeb vs. Mancozeb-Chlorpyrifos & 1 & 1.148 & .325 & 12.472 & .000 & 3.152 \\
\hline & Chlorpyrifos vs. Chlorpyrifos-Lambda cyhalothrin Mixture & 1 & .225 & .251 & .809 & .368 & 1.253 \\
\hline & Atrazine vs. Atrazine-Lambda-cyhalothrin & 1 & .828 & .274 & 9.109 & .003 & 2.289 \\
\hline & Mancozeb vs. Mancozeb-Lambda cyhalothrin & 1 & .919 & .296 & 9.646 & .002 & 2.507 \\
\hline & Atrazine vs. Atrazine-Chlorpyrifos & 1 & .634 & .258 & 6.009 & .014 & 1.884 \\
\hline & Atrazine vs. Quaternary mixture & 1 & .463 & .247 & 3.516 & .061 & 1.588 \\
\hline \multirow[t]{7}{*}{ Tilapia } & Atrazine vs. Atrazine-Mancozeb & 1 & .146 & .318 & .210 & .647 & 1.157 \\
\hline & Mancozeb vs. Mancozeb-Chlorpyrifos & 1 & .085 & .320 & .070 & .792 & 1.088 \\
\hline & Chlorpyrifos vs. Chlorpyrifos-Lambda cyhalothrin Mixture & 1 & 1.895 & .275 & 47.421 & .000 & 6.653 \\
\hline & Atrazine vs. Atrazine-Lambda-cyhalothrin & 1 & -.290 & .285 & 1.031 & .310 & .748 \\
\hline & Mancozeb vs. Mancozeb-Lambda cyhalothrin & 1 & -.161 & .303 & .284 & .594 & .851 \\
\hline & Atrazine vs. Atrazine-Chlorpyrifos & 1 & -.473 & .277 & 2.918 & .088 & .623 \\
\hline & Atrazine vs. Quaternary mixture & 1 & .274 & .329 & .696 & .404 & 1.315 \\
\hline
\end{tabular}

${ }^{*}$ Hazard ratio constant with time; $\mathrm{Pr}>\mathrm{ChiSq}$ indicates statistical significance if $<0.05$

Pulse toxicity of $13.48 \mathrm{mg} / \mathrm{L}$ mancozeb-lambda cyhalothrin was associated with pulse length and fish weight. The likelihood of survival after 15, 30, and $45 \mathrm{~min}$ exposure increased by a factor of 0.069 (93.1\%), 0.261 (73.9\%), $0.311(68.9 \%)$, respectively compared to $60 \mathrm{~min}(p<0.05)$, while survival likelihood increased by (99.95\%) in fishes weighing $0.1 \mathrm{~g}$ more than another fish.

Pulse toxicity of $0.57 \mathrm{mg} / \mathrm{L}$ chlorpyrifos-lambda cyhalothrin was associated with species, 15 , and $30 \mathrm{~min}$ pulse and fish weight \& length $(p<0.05)$. The survival probability of African catfish was 5.563 times higher than Nile tilapia. The likelihood of survival after 15 and $30 \mathrm{~min}$ exposure increased by a factor of $0.067(93.3 \%)$ and 0.270 (73\%), respectively, compared to $60 \mathrm{~min}$ of pulse exposure $(p<0.05)$. Survival probability increased by a factor of 25.627 and 0.059 in fishes weighing $0.1 \mathrm{~g}$ more than another fish and having condition factor higher by 0.1 , respectively.

Pulse toxicity of $11.72 \mathrm{mg} / \mathrm{L}$ quaternary mixture was associated with species, 15 , and $30 \mathrm{~min}$ pulse, length, and condition factor $(p<0.05)$. The likelihood of death after 15 and $30 \mathrm{~min}$ exposure decreased by a factor of 0.371 $(62.9 \%)$ and $0.273(72.7 \%)$, respectively, compared to $60 \mathrm{~min}$ of pulse exposure $(p<0.05)$. The likelihood of survival was 2.05 and 30.75 times lower for fish longer than another fish by $1 \mathrm{~cm}$ and condition factor higher by 0.1 .

\section{Discussion}

Concentration and time are important factors that influence toxicity. The high pesticide concentrations used in this study are comparable to the maximum worst-case concentration typically assumed in the tier-one stage of exposure assessment. The use of high concentration in 
Table 5 Cox PH model summary of all predictors on survival of fingerlings

\begin{tabular}{|c|c|c|c|c|c|c|c|c|c|}
\hline \multirow[t]{2}{*}{ Pesticide } & & \multirow[t]{2}{*}{$\begin{array}{l}\text { Parameter } \\
\text { estimate }\end{array}$} & \multirow[t]{2}{*}{ SE } & \multirow[t]{2}{*}{ Chi-square } & \multirow[t]{2}{*}{$d f$} & \multirow[t]{2}{*}{$\mathrm{Pr}>\mathrm{ChiSq}$} & \multirow[t]{2}{*}{ Hazard ratio } & \multicolumn{2}{|c|}{$\begin{array}{l}95.0 \% \mathrm{Cl} \text { for the } \\
\text { hazard ratio }\end{array}$} \\
\hline & & & & & & & & Lower & Upper \\
\hline \multirow[t]{8}{*}{ Atrazine-Mancozeb } & Species & .245 & .978 & .063 & 1 & .802 & $1.278^{\mathrm{a}^{*}}$ & .188 & 8.685 \\
\hline & $15 \mathrm{~min}$ & -.483 & .572 & .713 & 1 & .398 & .617 & .201 & 1.894 \\
\hline & $30 \mathrm{~min}$ & -1.102 & .613 & 3.228 & 1 & .072 & .332 & .100 & 1.105 \\
\hline & $45 \mathrm{~min}$ & .156 & .522 & .089 & 1 & .766 & 1.168 & .420 & 3.250 \\
\hline & $60 \mathrm{~min}$ & & & 4.509 & 3 & .212 & & & \\
\hline & Weight & -5.220 & 2.737 & 3.636 & 1 & .057 & .005 & .000 & 1.157 \\
\hline & Length & 1.132 & 1.011 & 1.254 & 1 & .263 & 3.103 & .427 & 22.521 \\
\hline & Condition factor & 1.670 & .708 & 5.560 & 1 & .018 & 5.312 & 1.326 & 21.284 \\
\hline \multirow[t]{8}{*}{ Atrazine-Chlorpyrifos } & Species & .129 & .574 & .051 & 1 & .822 & $1.138^{\mathrm{a}+}$ & .369 & 3.504 \\
\hline & $15 \min$ & -1.165 & .410 & 8.083 & 1 & .004 & .312 & .140 & .696 \\
\hline & $30 \mathrm{~min}$ & -.696 & .385 & 3.267 & 1 & .071 & .499 & .234 & 1.061 \\
\hline & $45 \mathrm{~min}$ & -.530 & .365 & 2.101 & 1 & .147 & .589 & .288 & 1.205 \\
\hline & $60 \mathrm{~min}$ & & & 8.531 & 3 & .036 & & & \\
\hline & Weight & -4.661 & 1.844 & 6.387 & 1 & .011 & .009 & .000 & .351 \\
\hline & Length & 1.063 & .658 & 2.611 & 1 & .106 & 2.895 & .797 & 10.507 \\
\hline & Condition factor & 1.007 & .329 & 9.375 & 1 & .002 & 2.737 & 1.437 & 5.213 \\
\hline \multirow[t]{8}{*}{ Atrazine-Lambda cyhalothrin } & Species & .554 & .610 & .825 & 1 & .364 & $1.740^{\mathrm{a}+}$ & .526 & 5.751 \\
\hline & $15 \min$ & -2.117 & .504 & 17.650 & 1 & .000 & .120 & .045 & .323 \\
\hline & $30 \mathrm{~min}$ & -1.290 & .403 & 10.265 & 1 & .001 & .275 & .125 & .606 \\
\hline & $45 \mathrm{~min}$ & -.967 & .365 & 7.039 & 1 & .008 & .380 & .186 & .777 \\
\hline & $60 \mathrm{~min}$ & & & 23.436 & 3 & .000 & & & \\
\hline & Weight & -6.316 & 3.407 & 3.436 & 1 & .064 & .002 & .000 & 1.436 \\
\hline & Length & -.210 & .770 & .074 & 1 & .785 & .811 & .179 & 3.668 \\
\hline & Condition factor & -.021 & .413 & .003 & 1 & .960 & .979 & .436 & 2.200 \\
\hline \multirow[t]{8}{*}{ Mancozeb-Chlorpyrifos } & Species & -.566 & .540 & 1.098 & 1 & .295 & $.568^{b+}$ & .197 & 1.637 \\
\hline & $15 \min$ & -1.163 & .552 & 4.430 & 1 & .035 & .313 & .106 & .923 \\
\hline & $30 \mathrm{~min}$ & -.938 & .493 & 3.625 & 1 & .057 & .391 & .149 & 1.028 \\
\hline & $45 \mathrm{~min}$ & -.099 & .460 & .047 & 1 & .829 & .906 & .368 & 2.229 \\
\hline & $60 \mathrm{~min}$ & & & 6.799 & 3 & .079 & & & \\
\hline & Weight & -1.823 & 4.821 & .143 & 1 & .705 & .162 & .000 & 2050.017 \\
\hline & Length & -1.790 & 1.242 & 2.079 & 1 & .149 & .167 & .015 & 1.903 \\
\hline & Condition factor & -1.165 & .895 & 1.694 & 1 & .193 & .312 & .054 & 1.802 \\
\hline \multirow[t]{8}{*}{ Mancozeb-Lambda cyhalothrin } & Species & .537 & .701 & .587 & 1 & .444 & $1.711^{\mathrm{a}+}$ & .433 & 6.760 \\
\hline & $15 \min$ & -2.674 & .573 & 21.746 & 1 & .000 & .069 & .022 & .212 \\
\hline & $30 \mathrm{~min}$ & -1.342 & .456 & 8.657 & 1 & .003 & .261 & .107 & 639 \\
\hline & $45 \mathrm{~min}$ & -1.167 & .451 & 6.682 & 1 & .010 & .311 & .129 & .754 \\
\hline & $60 \mathrm{~min}$ & & & 23.575 & 3 & .000 & & & \\
\hline & Weight & -2.991 & 1.331 & 5.048 & 1 & .025 & .050 & .004 & .683 \\
\hline & Length & .541 & .651 & .691 & 1 & .406 & 1.718 & .480 & 6.150 \\
\hline & Condition factor & 1.692 & .649 & 6.785 & 1 & .009 & 5.428 & 1.520 & 19.385 \\
\hline
\end{tabular}


Table 5 (continued)

\begin{tabular}{|c|c|c|c|c|c|c|c|c|c|}
\hline \multirow[t]{2}{*}{ Pesticide } & & \multirow[t]{2}{*}{$\begin{array}{l}\text { Parameter } \\
\text { estimate }\end{array}$} & \multirow[t]{2}{*}{ SE } & \multirow[t]{2}{*}{ Chi-square } & \multirow[t]{2}{*}{$d f$} & \multirow[t]{2}{*}{ Pr $>$ ChiSq } & \multirow[t]{2}{*}{ Hazard ratio } & \multicolumn{2}{|c|}{$\begin{array}{l}95.0 \% \mathrm{Cl} \text { for the } \\
\text { hazard ratio }\end{array}$} \\
\hline & & & & & & & & Lower & Upper \\
\hline \multirow[t]{8}{*}{ Chlorpyrifos-Lambda cyhalothrin } & Species & 1.716 & .488 & 12.379 & 1 & .000 & $5.563^{\text {a\# }}$ & 2.139 & 14.472 \\
\hline & $15 \min$ & -2.696 & .475 & 32.190 & 1 & .000 & .067 & .027 & .171 \\
\hline & $30 \mathrm{~min}$ & -1.308 & .364 & 12.924 & 1 & .000 & .270 & .132 & .552 \\
\hline & $45 \mathrm{~min}$ & -.187 & .337 & .309 & 1 & .579 & .829 & .428 & 1.606 \\
\hline & $60 \mathrm{~min}$ & & & 38.745 & 3 & .000 & & & \\
\hline & Weight & 3.244 & .779 & 17.326 & 1 & .000 & 25.627 & 5.564 & 118.033 \\
\hline & Length & -2.825 & .591 & 22.856 & 1 & .000 & .059 & .019 & .189 \\
\hline & Condition factor & .302 & .483 & .392 & 1 & .531 & 1.353 & .525 & 3.485 \\
\hline \multirow[t]{8}{*}{ Quaternary } & Species & 2.779 & .691 & 16.162 & 1 & .000 & $16.099^{\mathrm{a} \#}$ & 4.154 & 62.396 \\
\hline & $15 \mathrm{~min}$ & -.990 & .478 & 4.284 & 1 & .038 & .371 & .145 & .949 \\
\hline & $30 \mathrm{~min}$ & -1.298 & .462 & 7.884 & 1 & .005 & .273 & .110 & .676 \\
\hline & $45 \mathrm{~min}$ & -.378 & .394 & .921 & 1 & .337 & .685 & .316 & 1.483 \\
\hline & $60 \mathrm{~min}$ & & & 10.245 & 3 & .017 & & & \\
\hline & Weight & -1.280 & .946 & 1.831 & 1 & .176 & .278 & .044 & 1.775 \\
\hline & Length & .719 & .389 & 3.418 & 1 & .064 & 2.053 & .958 & 4.402 \\
\hline & Condition factor & 3.426 & 619 & 30.678 & 1 & .000 & 30.748 & 9.148 & 103.347 \\
\hline
\end{tabular}

${ }^{a}$ Indicates increase in hazard ratio, while ${ }^{b}$ indicates a decrease in hazard ratio compared with when other predictors were held constant; ${ }^{*}$ indicates significant when other predators were held constant but no longer significant; ${ }^{+}$indicates not significant when other predators were held constant and not significant now; ${ }^{*}$ indicates not significant when other predators were held constant but now significant

pulse assessment adequately characterizes toxic effects and provides a basis for estimating impacts at the predicted environmental concentration (Peterson et al., 2001). Furthermore, aquatic organisms may be exposed to a toxicant for only a short time before a critical threshold is achieved, resulting in an adverse effect (Naddy et al., 2000). The current study results show that the negative impact of brief exposure to pesticides may be comparable to continuous exposure. The probability of tilapia surviving $60 \mathrm{~min}$ of short exposure to mancozeb-lambda cyhalothrin mixture was similar to constant exposure for $96 \mathrm{~h}$. This is consistent with an earlier study, which reported that pulse exposure to pesticides might be more hazardous than continuous exposure (Tucker \& Burton, 1999).

The classic graphical method used to study chemical interaction is the isobologram (Huang et al., 2019). However, a survival graph can show the interaction between components of a mixture (Kanu et al., 2021b). Chemical interaction could enhance the toxicity of a mixture more than the single chemical and thus decrease the survival probability of organisms exposed to the mixture. Conversely, chemical interaction could reduce mixture toxicity, thus increasing the survival probability of organisms exposed to the mixture. In the current study, the antagonistic interaction of the pesticide mixture in African catfish may explain why the survival probability of Nile tilapia exposed to atrazine-chlorpyrifos, atrazine-lambda cyhalothrin, mancozeb-lambda cyhalothrin, and chlorpyrifos-lambda cyhalothrin was lower than the African catfish. This study also shows that pesticide mixture interaction or lack off was species-dependent. Not all pesticide mixtures studied showed interaction of mixture components in Nile tilapia as chlorpyrifos-lambda cyhalothrin mixture was antagonistic. The same pesticide mixtures were antagonistic in African catfish except for chlorpyrifos-lambda cyhalothrin, which may not have interacted. Finfish vary in their ability to metabolize xenobiotics, as some are efficient metabolizers than others (González et al., 2009). As such, the toxico-dynamics difference in the fish species may explain the antagonism or lack of interaction observed in this study.

We expected the survival probability of African catfish to be higher than Nile tilapia, given that African catfish are supposedly more tolerant to stressors than Nile tilapia (see Kanu et al., 2021a). However, the results of this study negate our prediction of the responses of both species after brief exposure to the pesticide mixture. Our earlier study showed that Nile tilapia had a significantly higher survival probability than African catfish after $60 \mathrm{~min}$ of exposure to atrazine, mancozeb, and lambda-cyhalothrin (Kanu et al., 2021a). In the current study, the survival 
probability of African catfish was approximately 6 and 16 times lower than Nile tilapia after exposure to chlorpyrifos-lambda cyhalothrin and quaternary mixture. On the other hand, survival likelihoods after pulse exposure to other pesticide mixtures were similar. Peterson et al., (2001) reported that Calineuria californica was approximately 1,000-fold less sensitive to carbaryl than Cinygma sp. only after pulsed exposure but similar after $96 \mathrm{~h}$ of continuous exposure. Differential response in this study may be linked to the physicochemical characteristics of pesticide and differences in uptake rate, time to equilibration, fish morphology, and pesticide metabolism, as alluded in our previous report (Kanu et al., 2021a).

A decrease in survival probability of exposed fingerlings as pulse-exposure length increased is consistent with previous reports. Peterson et al., (2001) observed that the survival of $C$. californica and Cinygma sp. decreased as pulse exposure time to carbaryl increased. However, in this study, survival probability was similar irrespective of pulse length for African catfish and Nile tilapia exposed to some pesticide mixtures. Low pesticides uptake during pulse exposure may be the reason for this. Peterson et al., (2001) observed low mortality (50\% mortality was not reached) for Calineuria californica pulsed exposed to three concentrations of carbaryl-17.3, 173 , and $1730 \mathrm{mg} / \mathrm{L}$ carbaryl for 15, 30, and $60 \mathrm{~min}$. Fifty percent $(50 \%)$ mortality occurred only after exposure to $1730 \mathrm{mg} / \mathrm{L}$ carbaryl for $60 \mathrm{~min}$. The authors attributed the low mortality to low rates of uptake of carbaryl by $C$. californica despite the high concentrations.

Bigger fingerlings had more tolerance for stress than smaller fingerlings and the same for fish with higher condition factors, as shown by this study. Fish condition factor depicts the health status of the fish; as such healthier fish usually have high condition factor (Kanu \& Idowu, 2017). Newman and McCloskey (2000) reported that smaller fish were more sensitive to benzocaine than bigger fish. An increase in survival probability with increasing size and condition factor supports the individual tolerance concept (Ashauer \& Escher, 2010). However, this study also shows that pesticide pulse toxicity may be independent of fingerling size and condition factor. Fish weight and length did not influence the mortality pattern of African catfish and Nile tilapia mortality following exposure to atrazine-lambda cyhalothrin, mancozebchlorpyrifos mixture, and African catfish exposed to the quaternary mixture. Likewise, the likelihood of survival after pulse exposure to some pesticide mixture was independent of fish condition factor as fingerlings with lower condition factors had a higher probability of survival. As such, mortality occurred independently of fish size and condition factor, indicating stochastic process may have influenced fingerling survival.
In this study, pesticide mixture interaction was speciesdependent. Innate intrinsic and extrinsic deterministic factors and, to a limited extent, stochastic processes may have influenced the survival probability of African catfish, and Nile tilapia pulsed exposed to complex pesticide mixtures. These findings have general significance relative to conducting exposure assessment for realistic risk assessment of pesticides. Selection of test species for regulatory testing and exposure assessment is critical for accurate ecological risk assessment of pesticides. Test species chosen based on sensitivity established by continuous toxicity tests may underestimate or overestimate pesticide pulse toxicity and undermine their role to provide broad protection to other non-target organisms within the ecosystem. There may be a need for a pulse toxicity test to rank the relative susceptibility of aquatic biota to pulse exposure. Environmental toxicologists could take advantage of the statistical power of survival analysis if the time to death or any other event (the endpoint of interest) is recorded during the exposure assessment.

\section{Conclusions}

The disparity in the influence of species difference, size, pulse length, and condition factor on the survival of African catfish and Nile tilapia pulse-exposed to pesticide mixtures depict scenarios that may occur in the environment. Given that ecotoxicology's goal is to predict the toxicity of pollutants in the environment, pulse and repeated pulse exposure toxicity tests should be incorporated in exposure assessment to make pesticides exposure assessment more realistic.

\section{Abbreviations}

PH: Proportional hazard; SPSS: Statistical package for social sciences; SAS: Statistical Analysis System; LT: Lethal time; LC: Lethal concentration.

\section{Acknowledgements}

Not applicable

\section{Authors' contributions}

KCK conceptualized, performed the experiment and statistical analysis, and wrote the original draft. AAO supervised, reviewed, and edited the initial draft, while NHA provided resources, supervised, and edited. All authors read and approved the final manuscript.

\section{Funding}

This research received no specific funding.

\section{Availability of data and materials}

All data generated or analyzed during this study are included in this published article and its supplementary information files.

\section{Declarations}

Ethics approval and consent to participate

Approval was obtained from the Ethical Committee at the College of Medicine, the University of Lagos, to use the fish species for pesticide toxicity 
studies (CMUL/ACUREC/10/20/827). All the fishes were handled humanely in compliance with Directive 2010/63/EU on protecting animals used for scientific purposes and the International Council for Laboratory Animal Science (ICLAS) ethical guidelines.

\section{Consent for publication}

Not applicable.

\section{Competing interests}

The authors declare that they have no competing interests.

\section{Author details}

'Department of Environmental Management and Toxicology, Michael Okpara University of Agriculture, Umudike, Nigeria. ${ }^{2}$ Ecotoxicology Unit, Department of Zoology, University of Lagos, Akoka-Yaba, Nigeria.

Received: 29 June 2021 Accepted: 17 February 2022

Published online: 07 March 2022

\section{References}

American Public Health Association (APHA). (1985). Standard methods for the examination of water and wastewater (16th ed., pp. 800-819). APHA.

Andersen, T. H., Tjørnhøj, R., Wollenberger, L., Slothuus, T., \& Baun, A. (2006). Acute and chronic effects of pulse exposure of Daphnia magna to dimethoate and pirimicarb. Environmental Toxicology and Chemistry: An International Journal, 25(5), 1187-1195.

Ashauer, R., \& Escher, B. I. (2010). Advantages of toxicokinetic and toxicodynamic modelling in aquatic ecotoxicology and risk assessment. Journal of Environmental Monitoring, 12(11), 2056-2061.

Busacker, G. P., Adelman, I. R., \& Goolish, E. M. (1990). Growth. In C. B. Schreck \& P. B. Moyle (Eds.), Methods for fish biology (pp. 363-387). American Fisheries Society.

Chèvre, N., \& Vallotton, N. (2013). Pulse exposure in ecotoxicology. In J. F. Férard \& C. Blaise (Eds.), Encyclopedia of aquatic ecotoxicology. Springer. https:// doi.org/10.1007/978-94-007-5704-2_84

Cold, A., \& Forbes, V. E. (2004). Consequences of a short pulse of pesticide exposure for survival and reproduction of Gammarus pulex. Aquatic Toxicology, 67(3), 287-299.

Dennis, N., Tiede, K., \& Thompson, H. (2012). Repeated and multiple stress (exposure to pesticides) on aquatic organisms. www.efsa.europa.eu/publications. Accessed May 23, 2020.

Dixon, P. M., \& Newman, M. C. (1991). Analyzing toxicity data using statistical models for time-to-death: an introduction. In M. C. Newman \& A. W. Mclntosh (Eds.), Metal ecotoxicology, concepts and applications (pp. 203-242). Lewis Publishers Inc.

Ewing, R. (1999). Diminishing returns: Salmon decline and pesticides. Journal of pesticide reform: a publication of the Northwest Coalition for Alternatives to Pesticides (USA).

González, J. F., Reimschuessel, R., Shaikh, B., \& Kane, A. S. (2009). Kinetics of hepatic phase I and II biotransformation reactions in eight finfish species. Marine Environmental Research, 67(4-5), 183-188.

Huang, R.Y., Pei, L., Liu, Q., Chen, S., Dou, H., Shu, G., Yuan, Z.X., Lin, J., Peng, G., Zhang. W., \& Fu, H. (2019). Isobologram analysis: a comprehensive review of methodology and current research. Frontiers in Pharmacology, 10, 1222. https://doi.org/10.3389/fphar.2019.01222.

Kanu, K. C., \& Idowu, E. T. (2017). Health status of Chrysichthys nigrodigitatus in response to aquatic pollution in Epe, Lagos and Ologe Lagoons, Southwest Nigeria. Environmental and Experimental Biology, 15(2), 151-159.

Kanu, K. C., Otitoloju, A. A., \& Amaeze, N. H. (2021a). Assessment of the risk of death of Clarias gariepinus and Oreochromis niloticus pulse-exposed to selected agricultural pesticides. Science and Reports, 11, 14652. https://doi. org/10.1038/s41598-021-94262-w

Kanu, K. C., Otitoloju, A. A., \& Amaeze, N. H. (2021 b). Estimation of pesticide mixture interaction in Nile tilapia (Oreochromis niloticus) using survival analysis. African Journal of Aquatic Science. https://doi.org/10.2989/16085 914.2021.1989572

Kumar, M., Chand, R., \& Shah, K. (2018). Mycotoxins and pesticides: Toxicity and applications in food and feed. In J. Patra, G. Das, \& H. S. Shin (Eds.), Microbial biotechnology (pp. 207-252). Springer.
Naddy, R. B., Johnson, K. A., \& Klaine, S. J. (2000). Response of Daphnia magna to pulsed exposures of chlorpyrifos. Environmental Toxicology and Chemistry, 19, 423-431.

National Center for Biotechnology Information. (2021). PubChem compound summary for CID 2730, chlorpyrifos. https://pubchem.ncbi.nlm.nih.gov/ compound/Chlorpyrifos. Accessed May 24, 2021.

Newman, M. C., \& Aplin, M. S. (1992). Enhancing toxicity data interpretation and prediction of ecological risk with survival time modeling: An illustration using sodium chloride toxicity to mosquitofish (Gambusia holbrooki). Aquatic Toxicology, 23(2), 85-96.

Newman, M. C., \& McCloskey, J. T. (1996). Time-to-event analyses of ecotoxicity data. Ecotoxicology, 5(3), 187-196.

Newman, M. C., \& McCloskey, J.T. (2000). The individual tolerance concept is not the sole explanation for the probit dose-effect model. Environmental Toxicology and Chemistry: An International Journal, 19(2), 520-526.

Peterson, J. L., Jepson, P. C., \& Jenkins, J. J. (2001). Effect of varying pesticide exposure duration and concentration on the toxicity of carbaryl to two field-collected stream invertebrates, Calineuria californica (Plecoptera: Perlidae) and Cinygma sp. (Ephemeroptera: Heptageniidae). Environmental Toxicology and Chemistry: an International Journal, 20(10), 2215-2223.

Raghavendra, S. N., Raghu, H. S., Chaithra, C., \& Rajeshwara, A. N. (2020). Potency of mancozeb conjugated silver nanoparticles synthesized from goat, cow and buffalo urine against Colletotrichum gloeosporioides causing anthracnose disease. Nature Environment \& Pollution Technology, 19(3), 969-979.

Sumon, K. A., Rashid, H., Peeters, E. T., Bosma, R. H., \& Van den Brink, P. J. (2018). Environmental monitoring and risk assessment of organophosphate pesticides in aquatic ecosystems of north-west Bangladesh. Chemosphere, 206, 92-100.

Tomlin, C. (1994). The pesticide manual-10th edition. The British Crop Protection Council and The Royal Society of Chemistry.

Tucker, K. A., \& Burton, G. A. (1999). Assessment of nonpoint-source runoff in a stream using in situ and laboratory approaches. Environmental Toxicology and Chemistry., 18, 2797-2803.

Wang, Y., Cang, T., Yu, R., Wu, S., Liu, X., Chen, C., Wang, Q., \& Cai, L. (2016). Joint acute toxicity of the herbicide butachlor and three insecticides to the terrestrial earthworm, Eisenia fetida. Environmental Science and Pollution Research, 23(12), 11766-11776.

West Africa Agricultural Productivity Programme (WAAPP-NIGERIA). (2013). Report of a baseline study on status of use, registration and regulation of pesticides in Nigeria. http://waapp.gov.ng/images/finalversionofbaselinestu diesonpestticideusesubmiitedtowaapp2013.pdf Accessed December 4, 2020

World Health Organization. (2003). Atrazine in drinking-water: background document for development of WHO guidelines for drinking-water quality (No. WHO/SDENSH/03.04/32). World Health Organization. Available from Microsoft Word-GDWQ.2ndEdit.Atrazine.doc (who.int). Accessed December 4, 2020

\section{Publisher's Note}

Springer Nature remains neutral with regard to jurisdictional claims in published maps and institutional affiliations. 